in the hematopoietic stem cell and progenitor cell compartment and in lifespan are closely linked at multiple loci in BXD recombinant inbred mice. Blood. 2004;104(2):374-379.

6. de Haan G, Nijhof W, Van Zant G. Mouse strain-dependent changes in frequency and proliferation of hematopoietic stem cells during aging: correlation between lifespan and cycling activity. Blood. 1997;89(5):1543-1550.

7. Geiger H, True JM, de Haan G, Van Zant G. Age- and stage-specific regulation patterns in the hematopoietic stem cell hierarchy. Blood. 2001;98(10):2966-2972.

8. de Haan G, Bystrykh LV, Weersing E, et al. A genetic and genomic analysis identifies a cluster of genes associated with hematopoietic cell turnover. Blood. 2002;100(6):2056-2062

9. Cahan P, Li Y, Izumi M, Graubert TA. The impact of copy number variation on local gene expression in mouse hematopoietic stem and progenitor cells. Nat Genet. 2009;41(4):430-437.

10. Liang Y, Jansen M, Aronow B, Geiger H, Van Zant G. The quantitative trait gene latexin influences the size of the hematopoietic stem cell population in mice. Nat Genet. 2007:39(2):178-188.

11. Zhang $C$, Fondufe-Mittendorf $Y N$, Wang $C$, et al. Latexin regulation by HMGB2 is required for hematopoietic stem cell maintenance. Haematologica. 2020;105(3):573-584

12. Ye ZJ, Kluger Y, Lian Z, Weissman SM. Two types of precursor cells in a multipotential hematopoietic cell line. Proc Natl Acad Sci U S A 2005;102(51):18461-18466.

13. You Y, Wen R, Pathak R, et al. Latexin sensitizes leukemogenic cells to gamma-irradiation-induced cell-cycle arrest and cell death through Rps3 pathway. Cell Death Dis. 2014;5:e1493.

14. Liu Y, Howard D, Rector K, et al. Latexin is down-regulated in hematopoietic malignancies and restoration of expression inhibits lymphoma growth. PLoS One. 2012;7(9):e44979.

15. Agresti A, Bianchi ME. HMGB proteins and gene expression. Curr Opin Genet Dev. 2003;13(2):170-178.

16. Aird KM, Iwasaki O, Kossenkov AV, et al. HMGB2 orchestrates the chromatin landscape of senescence-associated secretory phenotype gene loci. J Cell Biol. 2016;215(3):325-34.

\title{
Staying hydrated is important also for erythroblasts
}

\author{
Anupama Narla ${ }^{1}$ and Narla Mohandas ${ }^{2}$ \\ ${ }^{1}$ Department of Pediatrics, Stanford University, School of Medicine, Stanford, CA and ${ }^{2}$ Laboratory of Red Cell Physiology, New York \\ Blood Center, New York, NY, USA
}

E-mail: ANUPAMA NARLA - anunarla@stanford.edu or NARLAMOHANDAS - mnarla@nybc.org

doi:10.3324/haematol.2019.233999

I $\mathrm{n}$ this edition of Haematologica, Caulier and colleagues provide new insights into the role of PIEZO1, a mechanosensitive ion channel, in regulating normal human erythropoiesis. ${ }^{1}$ Defects in PIEZO1 have also been shown to lead to disordered erythropoiesis in hereditary xerocytosis, an inherited red cell disorder leading to red cell dehydration. ${ }^{2,3}$ Using in vitro cellular models of human erythropoiesis, the authors documented that the chemical activation of PIEZO1 either in an erythroid cell line model or in normal human hematopoietic stem cells (HSC) repressed erythroid differentiation. Importantly, they further documented that there was delayed erythroid differentiation in HSC from patients with PIEZO1 mutations. These findings provide unexpected and novel insights into the role of ion channels in the regulation of human erythropoiesis. ${ }^{1}$

Anemia is a significant health problem that affects nearly two billion people around the world. The major causes of anemia are: (i) an increased rate of destruction of circulating red cells in disorders that include red cell membrane disorders, sickle cell disease, immune hemolytic anemia, nutritional anemias and malaria; (ii) acute blood loss or splenic sequestration; and (iii) decreased production of red cells in the bone marrow due to ineffective erythropoiesis, which includes thalassemias, inherited bone marrow failure syndromes, infiltrative processes such as myelodysplastic syndrome and acute myeloid leukemia and suppression of erythropoiesis due to infection and medications. While significant progress has been made over the years to improve our understanding of the contribution of increased red cell destruction to anemia, much less is known about the extent of the effect of ineffective erythropoiesis and its contribution to anemia in the various red cell disorders. This is particularly true in the case of inherited red blood cell membrane disorders. The lack of progress has been due in part to a lack of an adequate and easily implementable methodology to study the complex process of human erythroid differentiation.

The generation of enucleated circulating human red cells is a complex biological process that begins in the bone marrow with the commitment of pluripotent HSC to the erythroid lineage (Figure 1). Subsequent stages of maturation include erythroid progenitors, burst-forming unit-erythroid and colony-forming unit-erythroid (CFUE), which can be identified by their development into representative clonal colonies of red cells in vitro. The CFU-E then undergoes terminal differentiation, progressing through four to five morphological stages, each having characteristic light microscopic and ultrastructural features. During terminal erythroid differentiation there is an increasing amount of hemoglobin synthesis accompanied by nuclear chromatin condensation and in the final stage of differentiation there is nuclear extrusion to generate an anucleate reticulocyte which over 2 to 3 days matures, first in the marrow and then in the circulation, into the discoid erythrocyte.

Significant progress has been made during the last decade in developing culture systems to study the differentiation of human CD34 cells intto enucleate reticulocytes and using various cell surface markers to monitor the progression through all stages of erythroid differentiation. ${ }^{4.7}$ These developments are enabling detailed characterization of normal and disordered human erythropoiesis. ${ }^{8-13}$ Importantly, as a result of this progress it is now possible to obtain insights into at what stage of the complex process of erythroid differentiation various genes contribute to ineffective erythropoiesis.

Using these in vitro cellular models of human erythropoiesis, the study by Caulier and colleagues documented that the chemical activation of PIEZO1 either in an ery- 


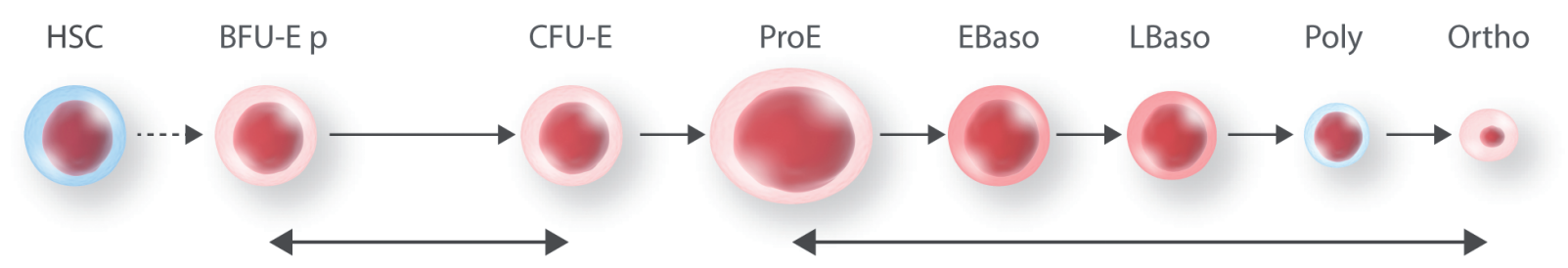

Erythroid progenitors

Terminal differentiation

Figure 1. Schematic representation of the various developmental stages of hematopoietic stem cells during erythroid differentiation. The multipotent hematopoietic stem cell first commits to the erythroid lineage to generate erythroid progenitors, which are recognized by their ability to form erythroid colonies in a semisolid methylcellulose culture system in response to interleukin-3, stem cell factor and erythropoietin. They cannot be distinguished based on their morphology. It is estimated that hematopoietic stem cells undergo approximately eight to ten cell divisions prior to the generation of the first morphologically recognizable erythroid cell in the bone marrow, the proerythroblast. During terminal erythroid differentiation, the proerythroblast undergoes five mitoses to generate an orthochromatic erythroblast. This ordered progression during normal erythropoiesis may be disturbed at any of the different developmental stages in various pathological states, leading to ineffective erythropoiesis. HSC: hematopoietic stem cell; BFU-E: burst-forming unit-erythroid; CFU-E: colony-forming unit-erythroid; ProE: proerythroblast; EBaso: early basophilic erythroblast; LBaso: late basophilic erythroblast; Poly: polychromatic erythroblast; Ortho: orthochromatic erythroblast.

throid cell line model or in primary normal HSC repressed erythroid differentiation. Importantly, the authors also showed that there was delayed erythroid differentiation of HSC from patients carrying PIEZO1 mutations. Delayed erythroid differentiation due to PIEZO1 activation was shown to be dependent on calcium entry and transcriptional control through the phosphorylation of transcription factors NFAT, STAT5 and ERK1/2.

These findings provide unexpected and novel insights into the role of ion channels in regulating human erythropoiesis. Although the reported findings represent an important step in our understanding of the role of PIEZO1 in regulating human erythropoiesis and ineffective erythropoiesis in hereditary xerocytosis, a number of questions remain unanswered. The variability in the delayed erythoid differentiation among different patients has not been defined. Furthermore, while ineffective erythropoiesis has been documented to be a feature of terminally differentiating erythroblasts, it is less clear at what specific stage of terminal erythroid differentiation apoptosis dominates. There is also no information about whether ineffective erythropoiesis is a feature of erythroid progenitors. It is anticipated that these important issues will be pursued in future studies.

In spite of some of these unanswered questions, the studies by Caulier and colleagues are significant in that they provide new and previously unsuspected insights into the role of ion channels in regulating human erythropoiesis. These valuable insights expand our current understanding of the role not only of growth factors and cytokines but also of ion channels in human erythroid differentiation. It is likely that the experimental strategies used in the study will be useful in furthering our understanding of the regulation of human erythropoiesis in general and the contribution of ineffective erythropoiesis to anemia in various human red cell disorders.

\section{References}

1. Caulier A, Jankovsky N, Demont Y, et al. PIEZO1 activation delays erythroid differentiation of normal and hereditary xerocytosis-derived human progenitor cells. Haematologica. 2020;105(3):610-622.

2. Zarychanski R, Schulz VP, Houston BL, et al. Mutations in the mechanotransduction protein PIEZO1 are associated with hereditary xerocytosis. Blood. 2017;120(9):1908-1915.

3. Picard V, Guitton C, Thuret I, et al. Clinical and biological features in PIEZO1-hereditary xerocytosis and Gardos-channelopathy: a retrospective series of 126 patients. Haematologica. 2019;104(8):15541564.

4. Anstee DJ, Gampel A, Toye AM. Ex-vivo generation of human red cells for transfusion. Curr Opin Hematol. 2012;19(3):163-169.

5. Hu J, Liu J, Xue F, et al. Isolation and functional characterization of human erythroblasts at distinct stages: implications for understanding of normal and disordered erythropoiesis in vivo. Blood. 2013;121(16):3246-3253

6. Li J, Hale J, Bhagia P, et al. Isolation and transcriptome analysis of human erythroid progenitors. Blood. 2014;124(24):3636-3645.

7. Yan H, Hale J, Jaffray J, et al. Developmental differences between neonatal and adult human erythropoiesis. Am J Hematol. 2018;93(4):494-503.

8. Liang L, Peng Y, Zhang J, et al. Deubiquitylase USP7 regulates human terminal erythroid differentiation by stabilizing GATA1. Haematologica. 2019;104(11):2178-2188.

9. Yan $\mathrm{H}$, Wang $\mathrm{Y}, \mathrm{Qu} \mathrm{X}$, et al. Distinct roles for TET family proteins in regulating human erythropoiesis. Blood. 2017;129(14): 2002-2012.

10. Rio S, Gastou M, Karboul N, et al. Regulation of globin-heme balance in Diamond-Blackfan anemia by HSP70/GATA1. Blood. 2019;133(12):1358-1370.

11. Ali AM, Huang Y, Pinheiro RF, et al. Severely impaired terminal erythrod differentiation as an independent prognostic marker in myelodysplastic syndromes. Blood Adv. 2018;2(12):1393-1402.

12. Gastou M, Rio S, Dussiot $M$, et al. The severe phenotype of Diamond Blackfan anemia is modulated by heat shock protein 70 . Blood Adv. 2017;1(12):1959-1976.

13. Dulmovits BM, Appiah-Kubi AO, Papoin J, et al. Pomalidomide reverses $\gamma$-globin silencing through the transcriptional reprogramming of adult hematopoietic progenitors. Blood. 2016;127(11):1481-1492. 